\title{
DISCONJUGACY AND INTEGRAL INEQUALITIES
}

\author{
BY \\ ACHIM CLAUSING ${ }^{1}$
}

\begin{abstract}
The basic data in this paper are a disconjugate differential operator and an associated two-point boundary value problem.

These define in a natural way a cone of functions satisfying a differential inequality with respect to the operator. By using a result of P. W. Bates and G. B. Gustafson on the monotonicity properties of Green's kernels it is shown that such a cone has a compact convex base which is a Bauer simplex. This result is used to derive a variety of integral inequalities which include known inequalities of Frank and Pick, Levin and Steckin, Karlin and Ziegler, as well as several new ones.
\end{abstract}

1. Introduction. It is the purpose of this paper to establish some integral inequalities for real functions $f$, defined on a finite interval, which are positive with respect to a disconjugate linear differential operator $L$ and satisfy certain boundary conditions.

It has been known for a long time that integral inequalities for, say, concave functions are closely related to integral representations. This point was made explicit as early as in 1916 by Blaschke and Pick [2], whose paper complemented another one by Frank and Pick [5] which seems to be the earliest source for a converse to Hölder's inequality. Implicitly in their paper was contained the following inequality which is valid for positive concave functions $f_{1}, f_{2}$ :

$$
\frac{2}{3} \int_{0}^{1} f_{1}(x) d x \int_{0}^{1} f_{2}(x) d x \leqslant \int_{0}^{1} f_{1}(x) f_{2}(x) d x .
$$

Recently-at least with respect to the long history of the subject-Karlin and Ziegler [7] generalized this inequality to the product of more than two concave functions. Generalizations of this inequality will also be the main subject of the present paper.

Concavity is related to the differential operator $L=-d^{2} / d x^{2}$. It was noted already by Blaschke and Pick that their results remain valid if $-d^{2} / d x^{2}$ is replaced by a general Sturm-Liouville operator. Karlin and Ziegler too extended their inequality to functions defined by more general second order operators and, moreover, to certain higher order selfadjoint operators. The stimulus for the present paper came from the observation that in doing so they used [7, p. 289] the variation diminishing property of the Green's functions associated with their operators in a

\footnotetext{
Received by the editors June 13, 1979.

AMS (MOS) subject classifications (1970). Primary 26A86.

Key words and phrases. Disconjugate differential operator, differential inequality, monotonicity properties of Green's kernels, integral representation, integral inequality.

${ }^{1}$ This paper was written while the author was a fellow of the Max Kade Foundation.
} 
way that was reminiscent of an argument employed by Bates and Gustafson [1, p. 334] in order to find estimates for Green's kernels of general disconjugate operators. Independently, a similar argument was used by the author in connection with integral representations for generalized completely convex functions associated with $L=d^{n} / d x^{n}(n \geqslant 2)$. It therefore suggested itself to consider integral inequalities for functions which are positive with respect to a disconjugate differential operator $L$ of arbitrary order, and to do this by using an integral representation for the cone $P_{L}$ of all such functions.

In $\$ 2$ of this paper we will prove that $P_{L}$ always has a compact base $B_{L}$ whose extremal point set ex $B_{L}$ is homeomorphic to $[0,1]$. The key to this is a monotonicity property of $L$-positive functions (see Corollary 2.3 ).

In $\$ 3$ we show that the restriction of certain linear functionals on $B_{L}$, such as $f \mapsto \int_{a}^{b} f(x) g(x) d x\left(g \in B_{L}\right)$, to the set ex $B_{L} \simeq[0,1]$ have monotonicity properties which ensure that one (or both) of their extremal values is attained at the endpoints of $[0,1]$. This yields a variety of integral inequalities.

In $\$ 4$ we illustrate the method by considering some concrete cases.

We would like to stress the fact here instead of stating it repeatedly in the results that all integral inequalities that we obtain in $\$ \S 3$ and 4 are sharp as one sees immediately from the proofs.

All functions to be considered are real-valued. If terms like positive, increasing, concave, etc. are meant in the strict sense this will always be explicitly stated.

Finally we want to mention that the comparison of the integral of a product of functions with the product of their integrals, although under different and weaker restrictions on the functions, is also the subject of an inequality of Grüss. We refer to $[9, \S 2.13]$ for references to this and some related inequalities.

2. Function cones defined by differential inequalities. By $L$ we will denote an ordinary linear differential operator of order $n \geqslant 2$, defined on a compact interval:

$$
L=\sum_{i=0}^{n} p_{i}(x) D^{i} \quad\left(D=\frac{d}{d x}\right),
$$

with the restrictions that $p_{n}=1$ and $p_{i} \in C^{i}[a, b], 0 \leqslant i \leqslant n-1$, so that the adjoint operator $L^{*}$ of $L$ is at hand. Furthermore, we require $L$ to be disconjugate on $[a, b]$. This means that every nontrivial solution $y$ of $L y=0$ has less than $n$ zeros on $[a, b]$ (counting multiplicities) or, equivalently, that $L$ can be written as a product of real linear factors of order 1 :

$$
L=\left(D-r_{n}\right) \ldots\left(D-r_{1}\right) \quad\left(r_{i} \in C^{n-i}[a, b], i=1, \ldots, n\right) .
$$

A detailed presentation of the theory of disconjugate differential operators can be found in the monograph [4] of Coppel.

By fixing an integer $m, 1 \leqslant m \leqslant n-1$, we determine a two-point boundary value problem:

$$
\begin{array}{ll}
L y=f & (f \in C[a, b]), \\
y^{(i)}(a)=y^{(j)}(b)=0 & (0 \leqslant i \leqslant m-1,0 \leqslant j \leqslant n-m-1) .
\end{array}
$$


The disconjugacy of $L$ entails that its solution can be obtained by using a Green's kernel $G(x, t)$ associated with $L, m: y(x)=\int_{a}^{b} G(x, t) f(t) d t$. We define functions $G_{t}$ by $G_{t}(x)=G(x, t)$ and recall that, for $t \in(a, b), G_{t}$ is in $C^{n-2}[a, b]$, satisfies the boundary conditions $G_{t}^{(i)}(a)=G_{t}^{(j)}(b)=0(0 \leqslant i \leqslant m-1,0 \leqslant j \leqslant n-m-1)$, and the equality $L G_{t}=\delta_{t}$ holds in the sense of distributions, $\delta_{t}$ being the Dirac measure at the point $t$.

By disconjugacy of $L, G$ has no zeros on $(a, b) \times(a, b)$ and $(-1)^{n-m} G(x, t) \geqslant 0$ [4, p. 108].

Frequently, we will make use of the fact that the Green's kernel $G^{*}(x, t)$ for the adjoint boundary value problem

$$
\begin{aligned}
& L^{*} y=f, \\
& y^{(i)}(a)=y^{(j)}(b)=0 \quad(0 \leqslant i \leqslant n-m-1,0 \leqslant j \leqslant m-1)
\end{aligned}
$$

is given by $G^{*}(x, t)=(-1)^{n} G(t, x)$. (Cf. [6]. We have assumed the leading coefficient of $L^{*}$ to be 1.) Note that as $L$ is disconjugate, so is $L^{*}[4$, p. 104]. The integral operator associated with $G$ will be denoted by $K$ :

$$
K f(x)=\int_{a}^{b} G(x, t) f(t) d t \quad(f \in C[a, b]) .
$$

$K$ is inverse to $L$ in the sense that $f=L K f$ for all $f \in C[a, b]$ and $f=K L f$ for all $f \in C^{n}[a, b]$ such that $f^{(i)}(a)=f^{(j)}(b)=0$ for $0 \leqslant i \leqslant m-1,0<j \leqslant n-m-1$.

For a $\sigma$-finite Radon measure $\mu$ on $(a, b)$ we put $K \mu(x)=\int_{a}^{b} G(x, t) d \mu(t)$. The operator $K^{*}$ is defined analogously using $G^{*}$.

Our main object of study will be the cone

$$
\begin{aligned}
Q_{L}=\left\{f \in C^{n}[a, b] \mid(-1)^{n-m} L f>0, f^{(i)}(a)=f^{(j)}(b)=0,\right. \\
0 \leqslant i \leqslant m-1,0 \leqslant j \leqslant n-m-1\},
\end{aligned}
$$

resp. its closure $P_{L}=\bar{Q}_{L}$, taken in the topology of locally uniform convergence on $(a, b)$. The sign in this definition is chosen to have all functions in $P_{L}$ positive.

For $t \in(a, b)$, the functions $(-1)^{n-m} G_{t}$ are contained in $P_{L}$. To verify this we choose a sequence $\left(\varphi_{k}\right)$ of positive continuous functions on $[a, b]$ such that $\varphi_{k}$ is supported by $[t-1 / k, t+1 / k]$ and $\left\|\varphi_{k}\right\|_{1}=1$. Then $(-1)^{n-m} K \varphi_{k} \in Q_{L}$, and this sequence converges uniformly to $(-1)^{n-m} G_{t}$ on compact subsets of $(a, b)$ as one easily checks.

Now we fix a nontrivial, bounded, positive Radon measure $\gamma$ on $(a, b)$. The following set will turn out to be a compact base for $P_{L}$ :

$$
B_{L}=\left\{f \in P_{L} \mid \gamma(f)=1\right\}
$$

For $t \in(a, b)$, we let $\tilde{G}_{t}=G_{t} / \gamma\left(G_{t}\right) \in B_{L}$. For later use, we introduce the function

$$
\begin{aligned}
\gamma^{*}(t) & =(-1)^{n-m} \gamma\left(G_{t}\right)=(-1)^{n-m} \int_{a}^{b} G(x, t) d \gamma(x) \\
& =(-1)^{m} \int_{a}^{b} G^{*}(t, x) d \gamma(x)=(-1)^{m} K^{*} \gamma(t) \quad(t \in[a, b]) .
\end{aligned}
$$

Whereas $G(x, t)=0$ on the boundary of $[a, b] \times[a, b]$, the $\operatorname{limits} \lim _{t \uparrow b} \tilde{G}_{t}$ and $\lim _{t \downarrow a} \tilde{G}_{t}$ exist in $B_{L}$. They will turn out to coincide with the two functions $v, w$ 
discussed by Bates and Gustafson [1], who used them to obtain estimates for the kernel $G(x, t)$.

The functions $v, w$ and their adjoint counterparts $w^{*}$ and $v^{*}$ are in $C^{n}[a, b]$ and are determined by these properties:

$$
\begin{array}{cc}
L v=0, & v^{(i)}(a)=v^{(j)}(b)=0,0 \leqslant i \leqslant m-1,0 \leqslant j \leqslant n-m-2, \gamma(v)=1, \\
L w=0, & w^{(i)}(a)=w^{(j)}(b)=0,0 \leqslant i \leqslant m-2,0 \leqslant j \leqslant n-m-1, \gamma(w)=1, \\
L^{*} w^{*}=0, & w^{*(i)}(a)=w^{*(j)}(b)=0, \\
& 0 \leqslant i \leqslant n-m-1,0 \leqslant j \leqslant m-2, \gamma\left(w^{*}\right)=1, \\
L^{*} v^{*}=0, & v^{*(i)}(a)=v^{*(j)}(b)=0, \\
& 0 \leqslant i \leqslant n-m-2,0 \leqslant j \leqslant m-1, \gamma\left(v^{*}\right)=1 .
\end{array}
$$

(The normalization here is different from that in [1].)

By disconjugacy of $L$ and $L^{*}$, none of these functions has a zero in $(a, b)$. Hence they are all positive.

We summarize those results of [1] that we need in the sequel. The notation

$$
k_{v}(x, t)=\frac{(-1)^{n-m} G(x, t)}{v(x) v^{*}(t)}, \quad k_{w}(x, t)=\frac{(-1)^{n-m} G(x, t)}{w(x) w^{*}(t)}
$$

$(x, t \in(a, b))$ will be convenient.

LemMa 2.1 (BATES-Gustafson). For $t \in(a, b), k_{v}(x, t)$ is a decreasing function of $x, \lim _{x \downarrow a} k_{v}(x, t)$ is finite and independent of $t$. Likewise, $k_{w}(x, t)$ is an increasing function of $x, \lim _{x \uparrow b} k_{w}(x, t)$ is finite and independent of $t$.

Proof. The monotonicity of $k_{v}(\cdot, t)$ is proved in [1, Lemma 3.4] (infra, cf. also [6, Theorem 2.1 1 ). As $k_{v} \geqslant 0$ and

$$
\lim _{x \uparrow b} k_{v}(x, t)=(-1)^{n-m} G_{t}^{(n-m-1)}(b) / v^{(n-m-1)}(b) v^{*}(t)=0,
$$

$k_{v}(\cdot, t)$ is decreasing. Note that $v^{(n-m-1)}(b) \neq 0$ by disconjugacy of $L$.

For the statement concerning $\lim _{x \downarrow a} k_{v}(x, t)$ see [1, Lemma 3.2]. The other half of the lemma holds by symmetry reasons.

Corollary 2.2. If $x \in(a, b)$, then

$$
\lim _{t \downarrow a} \tilde{G}_{t}(x)=w(x), \quad \lim _{t \uparrow b} \tilde{G}_{t}(x)=v(x) .
$$

Proof. We prove only the first statement. If we apply the above lemma to $G^{*}$ instead of $G$ then $w^{*}$ plays the role of $v$; hence $\lim _{t \downarrow a}(-1)^{m} G^{*}(t, x) / w^{*}(t) w(x)$ equals some constant which is independent of $x$. Furthermore, $\lim _{t \downarrow a} w^{*}(t) / \gamma^{*}(t)$ $=w^{*(n-m)}(a) / \gamma^{*(n-m)}(a)$; therefore the product of both limits exists and is constant:

$$
\lim _{t \downarrow a} \frac{(-1)^{m} G^{*}(t, x)}{w(x) \gamma^{*}(t)}=\lim _{t \downarrow a} \frac{\tilde{G}_{t}(x)}{w(x)}=c .
$$

As $\left\|G_{t}\right\|_{1}=\|w\|_{1}=1, c=1$. 
Actually, the convergence is even uniform on compacta. This will follow from the fact that $B_{L}$ is compact, and the essential tool in proving this is provided by another corollary to the lemma of Bates-Gustafson. Cf. [3, Theorem 3.6] for a similar result.

Corollary 2.3. If $f \in P_{L}$, then $f v^{-1}$ is decreasing and $f w^{-1}$ is increasing on $(a, b)$.

Proof. It is sufficient to give a proof for $f \in Q_{L}$. In this case, $f(x)=K L f(x)=$ $\int_{a}^{b} G(x, t) L f(t) d t$, that is

$$
f(x) v^{-1}(x)=\int_{a}^{b} \frac{\left|G_{t}(x)\right|}{v(x)}(-1)^{n-m} L f(t) d t .
$$

As $\left|G_{t}\right| / v$ is decreasing and $(-1)^{n-m} L f \geqslant 0, f v^{-1}$ is also decreasing. The second statement follows analogously.

This corollary implies that each nontrivial $f \in P_{L}$ is strictly positive on $(a, b)$ : If $f(x)=0$ for some $x \in(a, b)$, then $f w^{-1}=0$ on $(a, x]$ and $f v^{-1}=0$ on $[x, b)$; hence $f=0$.

The next two results refer to the topology of uniform convergence on compact subsets of $(a, b)$ :

Proposition 2.4. Any pointwise bounded subset $B$ of $P_{L}$ is relatively compact.

Proof. We show that pointwise boundedness implies equicontinuity of $\boldsymbol{B}$. For any $f \in P_{L}$ and $x, y \in(a, b), x \leqslant y, f(x) / v(x) \geqslant f(y) / v(y)$ and $f(x) / w(x)<$ $f(y) / w(y)$ by Corollary 2.3 , i.e.

$$
\frac{v(x)-v(y)}{v(y)} \leqslant \frac{f(x)-f(y)}{f(y)} \leqslant \frac{w(x)-w(y)}{w(y)} .
$$

If $x \geqslant y$, the converse inequalities hold. Therefore the following holds for all $x, y \in(a, b)$ :

$$
|f(x)-f(y)| \leqslant f(y) \max \left(\frac{|v(x)-v(y)|}{v(y)}, \frac{|w(x)-w(y)|}{w(y)}\right) .
$$

Now let $\varepsilon>0, y \in(a, b)$, and $f(y)<c$ for all $f \in B$. We put $s=\min (v(y), w(y))$ $>0, \delta=\varepsilon s c^{-1}$, and choose a neighborhood $U$ of $y$ such that $|v(x)-v(y)|<\delta$ and $|w(x)-w(y)|<\delta$ for $x \in U$. Then, by the inequality above, $|f(x)-f(y)|<c s^{-1} \delta$ $=\varepsilon$ for $f \in B, x \in U$. Hence $B$ is equicontinuous and thus relatively compact by Ascoli's theorem.

Corollary 2.5. $B_{L}$ is a compact convex base for $P_{L}$.

Proof. Similarly as in the foregoing proof one sees that for any $x, y \in(a, b)$, $f \in P_{L}$,

$$
f(x) \geqslant f(y) \min \left(\frac{v(x)}{v(y)}, \frac{w(x)}{w(y)}\right)
$$

holds. Denoting $\min (v(x), w(x))$ by $u(x)$, this implies $f(x) \geqslant c\|f\|_{\infty} u(x)(x \in$ $(a, b))$, where $c=\|\max (v(x), w(x))\|_{\infty}^{-1}$. 
Hence for $f \in B_{L}, 1=\gamma(f) \geqslant c\|f\|_{\infty} \gamma(u)$, and as $\gamma(u)>0$ by the strict positivity of $u$ on $(a, b)$ we obtain that $B_{L}$ is uniformly bounded on $[a, b]$. Proposition 2.4 yields $B_{L}$ to be compact. Also, $\gamma(f)>0$ for all $f \in P_{L} \backslash\{0\}$; thus $B_{L}$ is a base for $P_{L}$.

This corollary (or rather Proposition 2.4) shows that on $P_{L}$ the topologies of locally uniform and of pointwise convergence on $(a, b)$ coincide.

In particular, the two limits in Corollary 2.2 are uniform on compact subsets of $(a, b)$ and hence $v$ and $w$ lie in $B_{L}$, a fact that we had not established as yet.

As a consequence, one has that the following class of functions is contained (and dense) in $P_{L}$ :

$$
\begin{array}{r}
R_{L}=\left\{f \in C^{n}[a, b] \mid(-1)^{n-m} L f \geqslant 0, f^{(i)}(a)=f^{(j)}(b)=0,\right. \\
0 \leqslant i \leqslant m-2,0 \leqslant j \leqslant n-m-2, \\
\left.f^{(m-1)}(a) \geqslant 0,(-1)^{n-m-1} f^{(n-m-1)}(b) \geqslant 0\right\} .
\end{array}
$$

This is immediate from $w^{(m-1)}(a)>0,(-1)^{n-m-1} v^{(n-m-1)}(b)>0$, and $v, w \in P_{L}$ $\cap \operatorname{ker} L$.

Before we proceed to describe the extremal structure of $B_{L}$, we remark that for any $\sigma$-finite Radon measure $\mu$ on $(a, b)$ the continuous function $g=K \mu$ on $(a, b)$ satisfies $L g=\mu$ in the sense of distributions.

This results from Lagrange's formula: Let $\varphi \in \mathcal{D}(a, b)$, then

$$
\begin{aligned}
(L g, \varphi) & =\left(g,(-1)^{n} L^{*} \varphi\right)=\int_{a}^{b} g(x)(-1)^{n} L^{*} \varphi(x) d x \\
& =\int_{a}^{b} \int_{a}^{b}(-1)^{n} G(x, t) L^{*} \varphi(x) d \mu(t) d x=\int_{a}^{b} \int_{a}^{b} G^{*}(t, x) L^{*} \varphi(x) d x d \mu(t) \\
& =\int_{a}^{b} K^{*} L^{*} \varphi(t) d \mu(t)=\int_{a}^{b} \varphi(t) d \mu(t)=(\mu, \varphi) .
\end{aligned}
$$

The notation $\tilde{G}_{a}=w, \tilde{G}_{b}=v$ will be useful.

Proposition 2.6. The base $B_{L}$ of $P_{L}$ is a metrizable Bauer simplex, its set ex $B_{L}$ of extremal points consists of the functions $\tilde{G}_{t}, t \in[a, b]$.

Proof. If $f \in Q_{L}$, then

$$
f(x)=K L f(x)=\int_{a}^{b} G(x, t) L f(t) d t=\int_{a}^{b} \tilde{G}_{t}(x) \gamma^{*}(t)(-1)^{n-m} L f(t) d t ;
$$

hence the closed convex hull of $E=\left\{\tilde{G}_{t} \mid t \in(a, b)\right\}$ is all of $B_{L}$. By Milmans converse to the Krein-Milman theorem [10, p. 9], ex $B_{L}$ is contained in $\bar{E}=\left\{\tilde{G}_{t} \mid\right.$ $t \in[a, b]\}$. Hence, identifying $\bar{E}$ with $[a, b]$, any $f \in B_{L}$ can be represented by a Radon probability measure $\mu_{f}$ on $[a, b]$ :

$$
\begin{aligned}
f(x) & =\int_{a}^{b} \tilde{G}_{t}(x) d \mu_{f}(t)=\int_{a}^{b}(-1)^{n-m} G(x, t) \frac{d \mu_{f}(t)}{\gamma^{*}(t)} \\
& =\mu_{f}(\{a\}) w(x)+\mu_{f}(\{b\}) v(x)+(-1)^{n-m} K \nu_{f}(x),
\end{aligned}
$$

where $\nu_{f}$ is the measure on $(a, b)$ defined by $d \nu_{f}(t)=d \mu_{f}(t) / \gamma^{*}(t)$. 
If $f=\tilde{G}_{t}, t \in(a, b)$, then by the remark preceding this proposition

$$
\delta_{t} / \gamma^{*}(t)=(-1)^{n-m} L \tilde{G}_{t}=L K \nu_{f}=\nu_{f} ;
$$

thus $\tilde{G}_{t}$ has the unique representing measure $\mu_{f}=\delta_{t}$ and is therefore extremal.

Likewise, if $f=w$ or $f=v$, we get $0=(-1)^{n-m} L f=\nu_{f}$, i.e., $f=\mu_{f}(\{a\}) w+$ $\mu_{f}(\{b\}) v$. As $v$ and $w$ are not proportional, both are in ex $B_{L}$.

By the same argument, the measure $\nu_{f}$ and hence also $\mu_{f}$ are uniquely determined for each $f \in B_{L}$. Therefore $B_{L}$ is a Bauer simplex with ex $B_{L}$ homeomorphic to $[a, b]$. This implies the metrizability of $B_{L}$.

3. Integral inequalities. As already indicated in the introduction, the various integral inequalities to be considered all rest on the circumstance that certain functionals on $B_{L}$ are $\cap$-shaped or $\cup$-shaped when restricted to ex $B_{L}=[a, b]$ and hence attain their extremal values at $\tilde{G}_{a}=w$ or $\tilde{G}_{b}=v$.

A function $f$ on $[a, b]$ is called (strictly) $\cap$-shaped, if there exists a $t \in[a, b]$ such that $f$ (strictly) increases on $[a, t]$ and (strictly) decreases on $[t, b]$. Similarly, $f$ is called $\cup$-shaped, if it first decreases and then increases.

Concerning this notion, the key result in our situation is Lemma 3.2 below. Its proof employs a result which in essence is due to G. Pólya [11] (cf. also [4, p. 94]).

Proposition 3.1. Let $f \in C[a, b]$. If $K f$ has $k$ zeros in $(a, b)$, then $f$ has at least $k$ zeros in $(a, b)$. If $f$ has exactly $k$ zeros in $(a, b)$, then $f$ and $(-1)^{n-m} K f$ have the same sign in a neighborhood of $x=a$.

Proof. By disconjugacy, $L$ may be written as

$$
L y=v_{1} \ldots v_{n} D v_{n}^{-1} D \ldots D v_{2}^{-1} D v_{1}^{-1} y
$$

where $v_{i} \in C^{n-i+1}[a, b], v_{i}>0(i=1, \ldots, n)\left[4\right.$, p. 91]. As $y_{0}=K f$ has $n$ boundary zeros and $k$ zeros in $(a, b)$, it follows from Rolle's theorem that $f=L y_{0}$ has at least $k$ zeros in $(a, b)$. If $f$ has exactly $k$ zeros in $(a, b)$ and $x_{i}$ is the smallest zero of $y_{i}=D v_{i}^{-1} D \ldots D v_{1}^{-1} y_{0}$ in $(a, b)$, then for $x \in[a, b], x<\min \left(x_{i} \mid 0 \leqslant i \leqslant\right.$ $n)$, there are no sign changes in the sequence $y_{0}(x), y_{1}(x), \ldots, y_{m}(x)$ and $n-m$ sign changes in the sequence $y_{m}(x), \ldots, y_{n}(x)$. This follows by contradiction, using Rolle's theorem again. Thus $f$ and $(-1)^{n-m} K f$ have the same sign near $x=a$.

LemmA 3.2. Let $f, g \in C[a, b]$ and $g>0$ on $(a, b)$. If $f / g$ is increasing on $(a, b)$ (resp. decreasing, $\cap$-shaped, $\cup$-shaped), then $K f / K g$ is also increasing (resp. decreasing, $\cap$-shaped, $\cup$-shaped).

Proof. We prove only the statement for $\cap$-shaped functions; the others follow similarly. As $f / g$ is the pointwise limit of strictly $\cap$-shaped functions, we may as well assume that $f / g$ itself is strictly $\cap$-shaped. Therefore $f / g$ attains each value $c \in \mathbf{R}$ at most twice in $(a, b)$, i.e., $h=f-c g$ has at most two zeros in $(a, b)$. By Proposition 3.1, $K h=K f-c K g$ also has not more than two zeros in $(a, b)$ for each $c \in \mathbf{R}$; thus $K f / K g$ is either $\cap$-shaped or $U$-shaped. If $f / g$ takes a value $c$ at two different points of $(a, b)$, then $f-c g<0$ near $a$ and therefore $(-1)^{n-m} K f-$ $c(-1)^{n-m} K g<0$ near $a$. As the operator $(-1)^{n-m} K$ is positive, we get $K f / K g<c$ near $a$, and we conclude that $K f / K g$ is $\cap$-shaped. 
It is useful to observe that the following slight extension of this lemma is also valid, which is proved by an obvious approximation argument:

If $\mu$ is a bounded Radon measure on $(a, b)$ for which there is a sequence of continuous functions $f_{n}$ on $[a, b]$ such that $f_{n} / g$ is increasing (resp. decreasing, $\cap$-shaped, $U$-shaped) and $\mu=\lim _{n \rightarrow \infty} f_{n}$ weakly in the distribution sense, then $K \mu / K g$ is also increasing (resp. decreasing, $\cap$-shaped, $U$-shaped).

A typical application of the lemma is the following result:

Theorem 3.3. Let $h \in L^{1}[a, b]$ and $g \in C[a, b], g>0$ on $(a, b)$. Put

$$
I(f)=\frac{\int_{a}^{b} f(x) h(x) d x}{\int_{a}^{b} f(x) g(x) d x} \quad \text { for } f \in P_{L}, f \neq 0,
$$

and define constants

$$
c_{1}=\int_{a}^{b} w(x) h(x) d x, \quad c_{2}=\int_{a}^{b} v(x) h(x) d x,
$$

where $v$ and $w$ are normalized using $\gamma(f)=\int_{a}^{b} f(x) g(x) d x$ (cf. §2). Then the following holds for $f \in P_{L}, f \neq 0$ :

(a) If $h / g$ is increasing, $c_{1} \leqslant I(f) \leqslant c_{2}$.

(b) If $h / g$ is decreasing, $c_{2} \leqslant I(f) \leqslant c_{1}$.

(c) If $h / g$ is $\cap$-shaped, $\min \left(c_{1}, c_{2}\right) \leqslant I(f) \leqslant \sup _{t \in[a, b]} K^{*} h(t) / K^{*} g(t)$.

(d) If $h / g$ is $U$-shaped, inf ${ }_{t \in[a, b]} K^{*} h(t) / K^{*} g(t) \leqslant I(f) \leqslant \max \left(c_{1}, c_{2}\right)$.

Proof. Notice that $\gamma^{*}=(-1)^{m} K^{*} g$. The extremal values of $I(f)$ are attained for some $f=\tilde{G}_{t}, t \in[a, b]$. We define a function $\tilde{I}$ on $[a, b]$ by

$$
\begin{aligned}
\tilde{I}(t) & =I\left(\tilde{G}_{t}\right)=\left(\gamma^{*}(t)\right)^{-1} \int_{a}^{b}(-1)^{n-m} G(x, t) h(x) d x \\
& =\left(K^{*} g(t)\right)^{-1} \int_{a}^{b} G^{*}(t, x) h(x) d x=\frac{K^{*} h(t)}{K^{*} g(t)},
\end{aligned}
$$

and see that if $h / g$ is increasing (resp. decreasing, $\cap$-shaped, or $U$-shaped), then $\tilde{I}$ inherits the same property (apply Lemma 3.2 and the subsequent remark to $K^{*}$ instead of $K$ ). By using $\tilde{G}_{a}=w$ and $\tilde{G}_{b}=v$ the proof follows at once.

CoROllary 3.4. If $v$ and $w$ are normalized by $\|v\|_{1}=\|w\|_{1}=1$, then for $f \in P_{L}$ such that $\|f\|_{1}=1$ and $t \in[a, b]$ the following holds:

(a) $\min (v(t), w(t)) \leqslant f(t) \leqslant\left\|G_{t}^{*} / K^{*} 1\right\|_{\infty}$.

(b) $\int_{a}^{t} v(x) d x \leqslant \int_{a}^{t} f(x) d x \leqslant \int_{a}^{t} w(x) d x$.

Proof. Choose $g=1$. Let $\left(\varphi_{k}\right)$ be an approximation of $\delta_{t}$ by continuous functions as described in $\S 2$, and assume $\varphi_{k}$ to be $\cap$-shaped for all $k$. Then choose $h=\varphi_{k}$ in Theorem 3.3(c) and let $k$ tend to infinity. This yields (a). For (b), put $h=\mathbf{1}_{[a, t]}$, the indicator function of $[a, t]$, in Theorem 3.3(b).

As an illustration, consider $L=D^{2}$ on $[0,1]$. One obtains that any function $f \in C^{1}[0,1]$ such that $f(0)=0, f(1)=1$, for which $f^{\prime}$ is concave and positive, satisfies

$$
t^{2} \leqslant f(t) \leqslant 2 t-t^{2} \quad(t \in[0,1])
$$


Numerous variants of the corollary are feasible. One has to replace $H(x, t)=$ $\mathbf{1}_{[a, t]}(x)$, which is a Green's kernel for $L=D$, by the Green's kernels of other differential operators. For instance, the monotonicity properties of the kernels in Lemma 2.1 or in the subsequent Lemma 3.5 might be employed here. We omit the details.

Before stating the next lemma, we recall (cf. the proof of Proposition 3.1) that $L$ has a factorization $L y=L_{0} D v_{1}^{-1} y$, where $v_{1} \in C^{n}[a, b], v_{1}>0$, and $L_{0}$ is a linear differential operator of order $n-1$. In general, this factorization is not unique, nor need $L_{0}$ to be disconjugate on $[a, b]$.

LEMMA 3.5. If for one such factorization of $L$ the operator $L_{0}$ is disconjugate on $[a, b]$, then $(-1)^{n-m} G_{t} / v_{1}$ is $\cap$-shaped for all $t \in[a, b]$.

Proof. In view of Corollary 2.2 we may suppose $t \in(a, b)$. Similarly as in $\$ 2$ we approximate $(-1)^{n-m} G_{t}$ by $g_{k}=(-1)^{n-m} K \psi_{k}$, were $\psi_{k} \in C[a, b], \psi_{k}>0,\left\|\psi_{k}\right\|_{1}=$ 1 and $\left|\psi_{k}(x)\right| \leqslant 1 / n$ if $|x-t| \geqslant 1 / n$. Now let $c \geqslant 0$ and assume that $g_{k}-c v_{1}$ has at least 3 zeros in $(a, b)$. Then

$$
h_{k}=\left(\left(g_{k}-c v_{1}\right) / v_{1}\right)^{\prime}=\left(g_{k} / v_{1}\right)^{\prime}
$$

has at least 2 zeros in $(a, b) . h_{k}$ also has $n-2$ boundary zeros; hence its number of zeros on $[a, b]$ exceeds the order $n-1$ of the disconjugate operator $L_{0}$. By $[4$, Proposition 7, p. 94], $L L_{0} h_{k}=L g_{k}=(-1)^{n-m_{k}}$ has at least one zero on $(a, b)$, a contradiction to $\psi_{k}>0$.

Therefore $g_{k} / v_{1}$ attains each value $c \geqslant 0$ at most twice in $(a, b)$, so it is either $\cap$ or $U$-shaped. As $g_{k} / v_{1}$ converges pointwise to $(-1)^{n-m} G_{t} / v_{1}$, this function is also either $\cap$ - or $\cup$-shaped. Because it is positive and has zeros at $x=a$ and $x=b$, it is $\cap$-shaped.

The case $v_{1}=1$ is of special interest. Sufficient for $L=L_{0} D$ is $L 1=0$, but still $L_{0}$ need not be disconjugate on $[a, b]$.

THEOREM 3.6(a). Let $L$ have a factorization as above, where $L_{0}$ is disconjugate on $[a, b]$. Then for all $f, g \in P_{L}$

$$
c \int_{a}^{b} f(x) d x \int_{a}^{b} g(x) d x \leqslant \int_{a}^{b} f(x) g(x) \frac{d x}{v_{1}(x)}
$$

holds, where $c=\min \left(\int_{a}^{b} v^{2}(x) d x, \int_{a}^{b} v(x) w(x) d x, \int_{a}^{b} w^{2}(x) d x\right)$ and $v, w$ are normalized by $\|v\|_{1}=\|w\|_{1}=1$. Moreover, if $v / v_{1}$ is increasing, then the minimum is attained by one of the two numbers to the right, and if $w / v_{1}$ is decreasing, it is attained by one of the two numbers to the left.

Proof. $B_{L}$ is to be defined by taking $\gamma(f)=\|f\|_{1}$. The bilinear functional $F(f, g)=\int_{a}^{b} f(x) g(x) v_{1}^{-1}(x) d x$ attains its extremal values over $B_{L} \times B_{L}$ if $f, g \in$ ex $B_{L}$, i.e., $f=\tilde{G}_{s}, g=\tilde{G}_{t}, s, t \in[a, b]$. Therefore we define a function $\tilde{F}$ on $[a, b] \times[a, b]$ by

$$
\tilde{F}(s, t)=F\left(\tilde{G}_{s}, \tilde{G}_{t}\right)=\left(\gamma^{*}(t)\right)^{-1} \int_{a}^{b}(-1)^{m} G^{*}(t, x) \frac{\tilde{G}_{s}(x)}{v_{1}(x)} d x=\frac{K^{*}\left(\tilde{G}_{s} v_{1}^{-1}\right)(t)}{K^{*} 1(t)}
$$


By Lemma 3.5, $\tilde{F}$ is $\cap$-shaped as a function of $t$ and, by symmetry reasons, also as a function of $s$. Hence $\tilde{F}$ attains its minimum if $s, t \in\{a, b\}$. Under the additional assumptions on $v$, resp. $w, F(v, \cdot)$ is increasing and $F(\cdot, w)$ is decreasing; hence $F(v, w) \leqslant F(v, v)$, resp. $F(v, w) \leqslant F(w, w)$. This proves the theorem.

By a slight variation of the argument, we get

THEOREM 3.6(b). Under the same hypothesis on $L$ as in Theorem 3.6(a),

$$
c \int_{a}^{b} f(x) v_{1}(x) d x \int_{a}^{b} g(x) v_{1}(x) d x \leqslant \int_{a}^{b} f(x) g(x) d x
$$

holds for all $f, g \in P_{L}$. The expression for $c$ is the same as that in Theorem 3.6(a), if $v$ and $w$ are normalized by $\int_{a}^{b} v(x) v_{1}(x) d x=\int_{a}^{b} w(x) v_{1}(x) d x=1$.

Proof. This time one has to choose $\gamma(f)=\int_{a}^{b} f(x) v_{1}(x) d x$. Then the proof goes exactly like the foregoing one, except that one obtains $\tilde{F}(s, t)=K^{*} \tilde{G}_{s}(t) / K^{*} v_{1}(t)$. Again, Lemma 3.5 yields the conclusion.

It is natural, particularly in view of [7, Theorem 3], to look at products with more than two factors. A direct generalization of Theorem 3.6 depends on the question whether products $\Pi_{i=1}^{p} \tilde{G}_{t_{i}} v_{1}^{-1}\left(t_{i} \in[a, b], p \geqslant 2\right)$ are $\cap$-shaped. While this is true for second order operators $L\left[7\right.$, p. 286] and also for $L=D^{3}$, we did not succeed in deciding the general case. However we have:

THEOREM 3.7. Let $p \geqslant 1$ be an integer. Then

$$
\prod_{i=1}^{p} \int_{a}^{b} f_{i}(x) v^{p-1}(x) d x \leqslant \int_{a}^{b} \prod_{i=1}^{p} f_{i}(x) d x \leqslant c \prod_{i=1}^{p} \int_{a}^{b} f_{i}(x) v^{p-1}(x) d x
$$

holds for all $f_{1}, \ldots, f_{p} \in P_{L}$, where $c=\int_{a}^{b} w^{p}(x) d x$, if $w$ and $v$ are normalized by $\int_{a}^{b} v^{p}(x) d x=\int_{a}^{b} w(x) v^{p-1}(x) d x=1$.

Proof. The pattern of the proof is the same as of those for Theorems 3.3 and 3.6. First we fix a base $B_{L}$ by choosing $\gamma(f)=\int_{a}^{b} f(x) v^{p-1}(x) d x$. The extremal values of the multilinear functional $F\left(f_{1}, \ldots, f_{p}\right)=\int_{a}^{b} \Pi_{i=1}^{p} f_{i}(x) d x$ over $\left(B_{L}\right)^{p}$ are attained if $f_{i}=\tilde{G}_{t_{i}}$ for some $t_{i} \in[a, b], i=1, \ldots, p$. Therefore we consider the functions $\tilde{F}_{i}$ on $[a, b]$ defined by $\tilde{F}_{i}(t)=\int_{a}^{b} P(x) \tilde{G}_{t}(x) d x$, where $P(x)=$ $\Pi_{j=1 ; j \neq i}^{p} \tilde{G}_{t_{j}}(x)$, and observe that

$$
\tilde{F}_{i}(t)=\int_{a}^{b} P(x) \frac{(-1)^{m} G^{*}(t, x)}{\gamma^{*}(t)} d x=\frac{K^{*} P(t)}{K^{*} v^{p-1}(t)}
$$

The function $P / v^{p-1}=\prod_{j=1 ; j \neq i}^{p} \tilde{G}_{t j} v^{-1}$ is decreasing according to Lemma 2.1; hence $\tilde{F}_{i}$ is also decreasing by Lemma 3.2. We conclude that

$$
1=F(v, \ldots, v) \leqslant F\left(f_{1}, \ldots, f_{p}\right) \leqslant F(w, \ldots, w)=\int_{0}^{1} w^{p}(x) d x=c
$$

for $f_{1}, \ldots, f_{p} \in B_{L}$.

4. Examples. If in a concrete case one wishes to compute the constants in the inequalities above, the first thing to do is to check $L$ for being disconjugate on $[a, b]$. This is not easy in general, but it can be done in the examples below by direct inspection of the solutions of $L y=0$. 
The functions $v$ and $w$ can often be guessed. Usually they come first in nonnormalized form; then we denote them by $V$ and $W$.

EXAmple 4.1. $L=D^{n}(n \geqslant 2)$. This operator is clearly disconjugate on any interval; we choose $[0,1]$. For $1 \leqslant m \leqslant n-1$, the cone $P_{L}$ is the closure of

$$
\begin{aligned}
& R_{L}=\left\{f \in C^{n}[0,1] \mid(-1)^{n-m} f^{(n)} \geqslant 0,\right. f^{(i)}(0)=f^{(j)}(1)=0 \\
&(0 \leqslant i \leqslant m-2,0 \leqslant j \leqslant n-m-2), \\
&\left.\quad f^{(m-1)}(0) \geqslant 0,(-1)^{n-m-1} f^{(n-m-1)}(1) \geqslant 0\right\} .
\end{aligned}
$$

For $n=2$, these are the positive, concave functions on $[0,1]$. Clearly, $V(x)=$ $x^{m}(1-x)^{n-m-1}, W(x)=x^{m-1}(1-x)^{n-m}$. The following identity will be used:

$$
\int_{0}^{1} x^{p}(1-x)^{q} d x=\frac{\Gamma(p+1) \Gamma(q+1)}{\Gamma(p+q+2)} \quad(p, q>0) .
$$

If $p, q$ are integers, this equals

$$
\frac{p ! q !}{(p+q+1) !}=\left(\left(\begin{array}{c}
p+q \\
q
\end{array}\right)(p+q+1)\right)^{-1} \text {. }
$$

Choosing $h(x)=x^{\alpha}(\alpha>0)$ and $g(x)=1$ in Theorem 3.3(a) we obtain 4.1(a). If $f \in P_{L}$, then

$$
c_{1} \int_{0}^{1} f(x) d x \leqslant \int_{0}^{1} f(x) x^{\alpha} d x \leqslant c_{2} \int_{0}^{1} f(x) d x
$$

where

$$
c_{1}=n\left(\begin{array}{c}
n-1 \\
m-1
\end{array}\right)(n-m) ! \frac{\Gamma(m+\alpha)}{\Gamma(n+\alpha+1)}
$$

and

$$
c_{2}=n\left(\begin{array}{c}
n-1 \\
m
\end{array}\right)(n-m-1) ! \frac{\Gamma(m+\alpha+1)}{\Gamma(n+\alpha+1)} .
$$

In the case $n=2$, Theorem 3.3(c) yields an inequality which is partly known:

4.1(b). If $f$ is concave and positive on $[0,1]$ and $h$ is $\cap$-shaped and symmetric (i.e., $h(x)=h(1-x)$ ), then

$$
\int_{0}^{1} h(x) d x \int_{0}^{1} f(x) d x \leqslant \int_{0}^{1} f(x) h(x) d x \leqslant \int_{0}^{1} \hat{h}(x) d x \int_{0}^{1} f(x) d x
$$

where $\hat{h}$ is defined by $\hat{h}(x)=4 \min (x, 1-x) h(x)$. (The left half of the inequality is due to Levin and Stečkin [8, p. 3]. For this part of the result, positivity of $f$ is unnecessary, as can be seen by adding a suitable constant to $f$.)

Proof. We have $v(x)=2 x$. As $h$ is symmetric, $c_{1}=c_{2}$ in Theorem 3.3(c); thus

$$
\int_{0}^{1} 2 x h(x) d x \int_{0}^{1} f(x) d x \leqslant \int_{0}^{1} f(x) h(x) d x
$$

for concave, positive $f$. The symmetry of $h$ also implies $\int_{0}^{1} 2 x h(x) d x=\int_{0}^{1} h(x) d x$. To prove the right-hand side of the inequality, we have to compute $\sup _{t \in[0,1]} K^{*} h(t) / K^{*} 1(t)$. But $K^{*}=K$ has the kernel

$$
G(x, t)= \begin{cases}-x(1-t) & (0 \leqslant x \leqslant t \leqslant 1) \\ -t(1-x) & (0 \leqslant t \leqslant x \leqslant 1) .\end{cases}
$$


Therefore $K^{*} h / K^{*} 1$ is a symmetric, $\cap$-shaped function and thus attains its supremum at the point $x=\frac{1}{2}$. Using the kernel $G(x, t)$ we have $K^{*} h\left(\frac{1}{2}\right) / K^{*} 1\left(\frac{1}{2}\right)=$ $\int_{0}^{1} \hat{h}(x) d x$.

Particular choices of $h$ result in

$$
\frac{1}{6} \int_{0}^{1} f(x) d x \leqslant \int_{0}^{1} x(1-x) f(x) d x \leqslant \frac{5}{24} \int_{0}^{1} f(x) d x
$$

and

$$
\frac{2}{\pi} \int_{0}^{1} f(x) d x \leqslant \int_{0}^{1} f(x) \sin \pi x d x \leqslant \frac{8}{\pi^{2}} \int_{0}^{1} f(x) d x
$$

for all concave, positive functions $f$ (cf. $[8$, p. 5]). We return to the general case. It is obvious that $L=D^{n}$ satisfies the assumptions of Theorem 3.6 with $v_{1}=1$. Therefore we have:

4.1(c). For all $f, g \in P_{L}$,

$$
c \int_{0}^{1} f(x) d x \int_{0}^{1} g(x) d x \leqslant \int_{0}^{1} f(x) g(x) d x,
$$

where

$$
c=\frac{n^{2}}{2 n-1}\left(\begin{array}{c}
n-1 \\
m
\end{array}\right)\left(\begin{array}{c}
n-1 \\
m-1
\end{array}\right)\left(\begin{array}{c}
2 n-2 \\
2 m-1
\end{array}\right)^{-1} \text {. }
$$

Proof. With $c$ as just defined, we have to find the smallest of the three numbers $\int_{0}^{1} v(x) w(x) d x=c$,

$$
\begin{aligned}
& \int_{0}^{1} v^{2}(x) d x=\frac{n^{2}}{2 n-1}\left(\begin{array}{c}
n-1 \\
m
\end{array}\right)^{2}\left(\begin{array}{c}
2 n-2 \\
2 m
\end{array}\right)^{-1}=c \cdot \frac{2(n-m)}{2(n-m)-1}, \\
& \int_{0}^{1} w^{2}(x) d x=\frac{n^{2}}{2 n-1}\left(\begin{array}{c}
n-1 \\
m-1
\end{array}\right)^{2}\left(\begin{array}{c}
2 n-2 \\
2 m-2
\end{array}\right)^{-1}=c \cdot \frac{2 m}{2 m-1},
\end{aligned}
$$

from which the result manifestly follows.

For $n=2, m=1$ we get $c=\frac{2}{3}$, the constant mentioned in the introduction. The choice $n=4, m=2$ yields $c=36 / 35$. For this case the above inequality is also known [7, Theorems 6 and 7]. It might be interesting to compare that for $m=1$ one obtains $c=n^{2}(n-1) / 2(2 n-1)$, whereas $m=n-1$ yields

$$
c=n^{2} / 2(2 n-1) \text {. }
$$

Finally we want to illustrate the use of Theorem 3.7. We restrict ourselves to $n=2$.

4.1(d). Let $p \geqslant 1$ be an integer. Then

$$
\begin{aligned}
(p+1)^{p-1} \prod_{i=1}^{p} \int_{0}^{1} f_{i}(x) x^{p-1} d x & <\int_{0}^{1} \prod_{i=1}^{p} f_{i}(x) d x \\
& <p^{p}(p+1)^{p-1} \prod_{i=1}^{p} \int_{0}^{1} f_{1}(x) x^{p-1} d x
\end{aligned}
$$

holds for all concave, positive functions $f_{1}, \ldots, f_{p}$. 
Proof. The normalization of Theorem 3.7 yields $v(x)=(p+1)^{1 / p} x, w(x)=$ $p(p+1)^{1 / p}(1-x)$. Hence the constant in that theorem is $c=\int_{0}^{1} w^{p}(x) d x=p^{p}$.

If in 4.1(c) we replace the integration interval $[0,1]$ by $[0, b], b>0$, then we get another constant, say $c_{b}$, which is related to $c$ by $b c_{b}=c$. The quantity $(b-a) c$, where $c$ is the constant of Theorem 3.6 in the case $v_{1}=1$, has an obvious interpretation as a lower bound for the mean value of a product of two functions having mean value 1 . In the next example this quantity varies with the length of the interval.

EXAmple 4.2. $L=D^{3}-\operatorname{ctg} x D^{2}$. The factorization $L=L_{0} D$ with $L_{0}=D^{2}-$ $\operatorname{ctg} x D$ is immediate. A base for ker $L$ is given by $\{1, x, \sin x\}$ and one for $\operatorname{ker} L_{0}$ by $\{1, \cos x\}$. From this we see that $L$ and $L_{0}$ are disconjugate on $[\varepsilon, \pi-\varepsilon]$ for all $\varepsilon, 0<\varepsilon<\pi / 2$. We put $m=2$. Then

$$
W(x)=\sin x-\sin \varepsilon \text { and } V(x)=(x-\varepsilon) \cos \varepsilon-W(x) .
$$

The cone $P_{L}=P_{L}^{\varepsilon}$ is the closure of

$$
R_{L}^{\varepsilon}=\left\{f \in C^{3}[\varepsilon, \pi-\varepsilon] \mid f \geqslant 0, f^{\prime \prime} \operatorname{ctg} x \geqslant f^{\prime \prime \prime}, f^{\prime}(\varepsilon) \geqslant 0\right\} .
$$

4.2(a). For $f, g \in P_{L}^{\varepsilon}$ the following holds:

$$
c_{\varepsilon} \int_{\varepsilon}^{\pi-\varepsilon} f(x) d x \int_{\varepsilon}^{\pi-\varepsilon} g(x) d x \leqslant \int_{\varepsilon}^{\pi-\varepsilon} f(x) g(x) d x,
$$

where the constant $c_{\varepsilon}$ is given by the following formula, in which $\delta=\pi / 2-\varepsilon$ :

$c_{\varepsilon}=\left(\left(\frac{3}{2}-\delta^{2}\right) \sin 2 \delta-2 \delta \cos 2 \delta-\delta\right) / 4\left(\delta \cos \delta-\left(1-\delta^{2}\right) \sin \delta\right)(\sin \delta-\delta \cos \delta)$. In particular, for $d_{\varepsilon}=2 \delta c_{\varepsilon}$ we find

$$
\lim _{\varepsilon \downarrow 0} d_{\varepsilon}=\frac{\pi^{2}}{2\left(\pi^{2}-4\right)}=0.840 \ldots \text { and } \lim _{\varepsilon \uparrow \pi / 2} d_{\varepsilon}=\frac{9}{10} .
$$

Proof. $V$ is increasing, so we only have to find the smaller one of the numbers $c_{1}=\int_{\varepsilon}^{\pi-\varepsilon} v(x) w(x) d x$ and $c_{2}=\int_{\varepsilon}^{\pi-\varepsilon} w^{2}(x) d x, v$ and $w$ being normalized by $\|v\|_{1}=\|w\|_{1}=1$. The relevant integrals are

$$
\begin{aligned}
\int_{\varepsilon}^{\pi-\varepsilon} W(x) d x & =2(\sin \delta-\delta \cos \delta)=\frac{2}{3} \delta^{3}+O\left(\delta^{5}\right) \quad(\delta \rightarrow 0), \\
\int_{\varepsilon}^{\pi-\varepsilon} V(x) d x & =2\left(\delta \cos \delta-\left(1-\delta^{2}\right) \sin \delta\right)=\frac{4}{3} \delta^{3}+O\left(\delta^{5}\right) \quad(\delta \rightarrow 0), \\
\int_{\varepsilon}^{\pi-\varepsilon} W^{2}(x) d x & =2 \delta-\frac{3}{2} \sin 2 \delta+\delta \cos 2 \delta=\frac{4}{15} \delta^{5}+O\left(\delta^{7}\right) \quad(\delta \rightarrow 0), \\
\int_{\varepsilon}^{\pi-\varepsilon} V(x) W(x) d x & =-\delta+\left(\frac{3}{2}-\delta^{2}\right) \sin 2 \delta-2 \delta \cos 2 \delta \\
& =\frac{2}{5} \delta^{5}+O\left(\delta^{7}\right) \quad(\delta \rightarrow 0) .
\end{aligned}
$$

From this the result follows by some easy computations.

The next example behaves rather similarly to the foregoing one, except that now $L_{0}$ has a strictly smaller range of disconjugacy than $L$. 
Example 4.3. $L=D^{3}+D$. We write $L=L_{0} D$, where $L_{0}=D^{2}+1$. The kernels of $L$ and $L_{0}$ are spanned by $\{1, \sin x, \cos x\}$ and $\{\sin x, \cos x\}$, resp.; hence $L$, resp. $L_{0}$, is disconjugate on $[a, b]$ if and only if $b-a<2 \pi$, resp. $b-a<\pi$. We choose a symmetric integration interval $[-a, a], 0<2 a<\pi$, and put $m=1$. Then $P_{L}=P_{L}^{a}$ is the closure of

$$
R_{L}^{a}=\left\{f \in C^{3}[-a, a] \mid f \geqslant 0, f^{\prime \prime \prime}+f^{\prime} \geqslant 0, f^{\prime}(a) \leqslant 0\right\} .
$$

We find $W(x)=\cos x-\cos a$ and $V(x)=1-\cos (a-x)$, and using the integrals

$$
\begin{aligned}
\int_{-a}^{a} W(x) d x & =2 \sin a-2 a \cos a, \quad \int_{-a}^{a} V(x) d x=2 a-\sin 2 a, \\
\int_{-a}^{a} W^{2}(x) d x & =2 a-\frac{3}{2} \sin 2 a+a \cos 2 a \\
\int_{-a}^{a} V(x) W(x) d x & =\frac{9}{4} \sin a+\frac{1}{4} \sin 3 a-3 a \cdot \cos a,
\end{aligned}
$$

one verifies easily the following inequality:

$$
c_{a} \cdot \int_{-a}^{a} f(x) d x \int_{-a}^{a} g(x) d x \leqslant \int_{-a}^{a} f(x) g(x) d x \quad\left(f, g \in P_{L}^{a}\right), \quad \text { (4.3(a)) }
$$

where the constant $c_{a}$ is given by

$$
c_{a}=(9 \sin a+\sin 3 a-12 a \cdot \cos a) / 8(2 a-\sin 2 a)(\sin a-a \cdot \cos a) .
$$

In particular, for $d_{a}=2 a \cdot c_{a}$ we find

$$
\lim _{a \downarrow 0} d_{a}=\frac{9}{10}, \quad d_{\pi / 4}=0.922 \ldots, \quad \lim _{a \uparrow \pi / 2} d_{a}=1 .
$$

Our final example deals with a nonbounded integration interval:

EXAMPLE 4.4. $L=(D+1)^{n}(n \geqslant 2)$. The given factorization of $L$ shows that the operator is disconjugate on every interval. By writing $L y=e^{-x} D^{n}\left(e^{x} y\right)$ we find that the solutions of $L y=0$ are the functions $e^{-x} q(x)$, where $q$ is a polynomial of degree less than $n$. We take $[0, a], a>0$, as the basic interval and choose $m=n-1$. Clearly,

$$
V(x)=x^{n-1} e^{-x} \text { and } W(x)=x^{n-2}(a-x) e^{-x}=W_{a}(x) .
$$

$P_{L}$ is the closure of $R_{L}=R_{L, a}=\left\{f \in C^{n}[0, a] \mid f(x)>0\right.$ and $\left(e^{x} f\right)^{(n)}(x)<0$ for $\left.x \in[0, a], f^{(k)}(0)=0(k=0, \ldots, n-3), f^{(n-2)}(0) \geqslant 0\right\}$.

Furthermore we define $R=\bigcap_{a>0} R_{L, a}=\left\{f \in C^{n}[0, \infty) \mid f(x) \geqslant 0\right.$ and $\left(e^{x} f\right)^{(n)}(x) \leqslant 0$ for $\left.x \geqslant 0, f^{(k)}(0)=0(k=0, \ldots, n-3), f^{(n-2)}(0) \geqslant 0\right\}$.

4.4(a). For $f, g \in R$ the following inequality holds:

$$
\frac{(2 n-3) !}{(n-1) !(n-2) !} \cdot \int_{0}^{\infty} f(x) d x \int_{0}^{\infty} g(x) d x \leqslant \int_{0}^{\infty} f(x) g(x) e^{x} d x
$$

provided the integrals are finite.

Proof. If $f, g \in R$, then $\left.f\right|_{[0, a]}$ and $\left.g\right|_{[0, a]}$ are in $R_{L, a}(a>0)$, and hence

$$
\int_{0}^{\infty} f(x) g(x) e^{x} d x \geqslant \int_{0}^{a} f(x) g(x) e^{x} d x \geqslant c_{a} \cdot \int_{0}^{a} f(x) d x \int_{0}^{a} g(x) d x,
$$


where $c_{a}$ is the constant provided by Theorem 3.6(a), applied with $L_{0}=e^{-x} D^{n-1}$ and $v_{1}(x)=e^{-x}$. To compute $c_{a}$, we put

$$
\begin{aligned}
d_{a}= & \int_{0}^{a} V(x) W_{a}(x) e^{x} d x / \int_{0}^{a} V(x) d x \int_{0}^{a} W_{a}(x) d x \\
= & \int_{0}^{a} x^{2 n-3}(a-x) e^{-x} d x / \int_{0}^{a} x^{n-1} e^{-x} d x \int_{0}^{a} x^{n-2}(a-x) e^{-x} d x \\
= & \left(\int_{0}^{a} x^{2 n-3} e^{-x} d x-\frac{1}{a} \int_{0}^{a} x^{2 n-2} e^{-x} d x\right) / \\
& / \int_{0}^{a} x^{n-1} e^{-x} d x\left(\int_{0}^{a} x^{n-2} e^{-x} d x-\frac{1}{a} \int_{0}^{a} x^{n-1} e^{-x} d x\right) .
\end{aligned}
$$

From this we obtain that

$$
\lim _{a \rightarrow \infty} d_{a}=\frac{(2 n-3) !}{(n-1) !(n-2) !} .
$$

Likewise we put $e_{a}=\int_{0}^{a} W_{a}^{2}(x) e^{x} d x /\left(\int_{0}^{a} W_{a}(x) d x\right)^{2}$, and find

$$
\lim _{a \rightarrow \infty} e_{a}=\frac{(2 n-4) !}{((n-2) !)^{2}}
$$

Therefore $e_{a}>d_{a}$ at least for sufficiently large $a>0$, so the constant $c_{a}$ is equal to $d_{a}$. By letting $a$ tend to infinity in the inequality at the beginning of the proof, we obtain 4.4(a).

As the constants $c_{a}$ in this proof are sharp, one can prove that $4.4(a)$ is also a sharp inequality. We omit the details.

\section{REFERENCES}

1. P. W. Bates and G. B. Gustafson, Green's function inequalities for two-point boundary value problems, Pacific J. Math. 59 (1975), 327-343.

2. W. Blaschke and G. Pick, Distanzschätzungen im Funktionenraum. II, Math. Ann. 77 (1916), 277-300.

3. A. Clausing, Pólya's condition and polynomial expansions about two points (in preparation).

4. W. A. Coppel, Disconjugacy, Lecture Notes in Math., vol. 220, Springer-Verlag, Berlin and New York, 1971.

5. Ph. Frank and G. Pick, Distanzschätzungen im Funktionenraum. I, Math. Ann. 76 (1915), 354-375.

6. Ph. Hartman, Monotony properties and inequalities for Green's functions for multipoint boundary value problems, SIAM J. Math. Anal. 9 (1978), 806-814.

7. S. Karlin and Z. Ziegler, Some inequalities for generalized concave functions, J. Approximation Theory 13 (1975), 276-293.

8. V. I. Levin and S. B. Stečkin, Inequalities, Amer. Math. Soc. Transl. (2) 14 (1960), 1-29.

9. D. S. Mitrinovič, Analytic inequalities, Springer-Verlag, Berlin and New York, 1970.

10. R. R. Phelps, Lectures on Choquet's theorem, Van Nostrand-Reinhold, New York, 1966.

11. G. Polya, On the mean-value theorem corresponding to a given linear homogeneous differential equation, Trans. Amer. Math. Soc. 24 (1922), 312-324.

Department of Mathematics, University of Washington, Seattle, Washington 98195

Current address: Institut für Mathematische Statistik, Westfälische Wilhelms-Universität, D-4400 Münster, West Germany 